\title{
Improving Graph Matching via Density Maximization
}

\author{
Chao Wang, Lei Wang \\ School of Computer Science \& Software Engineering \\ University of Wollongong, NSW, Australia, 2522 \\ chaow, leiw@uow.edu.au
}

\author{
Lingqiao Liu \\ CECS, Australian National University \\ ACT 0200, Canberra, Australia \\ lingqiao.liu@cecs.anu.edu.au
}

\begin{abstract}
Graph matching has been widely used in various applications in computer vision due to its powerful performance. However, it poses three challenges to image sparse feature matching: (1) The combinatorial nature limits the size of the possible matches; (2) It is sensitive to outliers because the objective function prefers more matches; (3) It works poorly when handling many-to-many object correspondences, due to its assumption of one single cluster for each graph. In this paper, we address these problems with a unified framework-Density Maximization. We propose a graph density local estimator ( $D L E)$ to measure the quality of matches. Density Maximization aims to maximize the DLE values both locally and globally. The local maximization of DLE finds the clusters of nodes as well as eliminates the outliers. The global maximization of DLE efficiently refines the matches by exploring a much larger matching space. Our Density Maximization is orthogonal to specific graph matching algorithms. Experimental evaluation demonstrates that it significantly boosts the true matches and enables graph matching to handle both outliers and many-to-many object correspondences.
\end{abstract}

\section{Introduction}

Sparse feature correspondence (SFC) is a fundamental problem for a wide range of applications in computer vision, such as image retrieval, object recognition, 3D reconstruction, and motion estimation. Since these feature sets have meaningful internal structure, they are often considered as two separate graphs, but not simply as point sets. As a result, SFC can be modelled as graph matching in which graph nodes represent features extracted from each image while graph edges represent relationships between features. Graph matching finds a mapping between the two feature sets by minimizing the distortions of the two graphs. Compared to the parametric models (e.g. Thin-Plate Spline) and the methods with geometric constraints (e.g., RANSAC with rigid transformation assumption), graph matching pro- vides greater flexibility for object modeling and is more robust to large non-rigid transformations.

There have been a myriad of algorithms proposed for graph matching [7]. Those before 1990s did not aim to optimize a well-defined objective function. Among recent algorithms, the Integer Quadratic Programming (IQP) has emerged as a de facto formulation of graph matching $[2,8,14,15,23,19,11]$. IQP explicitly considers both unary and pair-wise terms which reflect the compatibilities in feature appearance as well as pair-wise geometric relationships. Since IQP is NP-complete, the optimal solution is virtually unachievable and approximations are required. While recent approximate methods have led to tremendous progress, the results for many real-world images are still far from being perfect due to several factors.

Aside from its NP-complete nature, IQP owns several limitations some of which might not have been explicitly pointed out before. Firstly, the combinatorial nature of graph matching makes computation of the full affinity matrix in IQP intractable for large graphs. Secondly, due to the non-negative property of the edge attributes, the objective function of IQP prefers more matches even if they are outliers. Last but not least, IQP assumes that each graph contains only one cluster of nodes. In real-world cases, however, image pairs can have a large number of sparse features, significant clutter, multiple objects, and even many-to-many object correspondences. Therefore, graph matching poses three challenges to SFC: (1) its combinatorial nature limits the size of the possible matches; (2) it is sensitive to outliers; (3) it works poorly for many-to-many object correspondences.

To address the first challenge, most methods establish the set of candidate matches by using unary descriptors of discriminative features, such as SIFT [18], at a relatively low cost. Then only a small number of matches are utilized to build an initial graph. Their results might be unsatisfactory due to the loss of useful information hidden in the full matching space [5]. Cho et al.[5] proposed a progressive framework to update candidate matches based on pair-wise geometric relationships between new matches and the cur- 
rent graph matching result. It greatly boosts the objective function of IQP. However, it tends to introduce many outliers because the current graph matching result might be noisy. Furthermore, its computational complexity is high because exploring the full matching space is required.

To address the second challenge, some popular attempts extend the general graph to the hyper-graph [9, 13, 24]. By using higher-order constraints (e.g., projective invariance) instead of the unary or pair-wise ones, such methods successfully filter out most outliers. Unfortunately, they also work poorly for many-to-many object correspondences due to the single cluster assumption.

To address both the second and the third challenges simultaneously, unsupervised clustering might be the most promising approach. Each cluster of matches naturally corresponds to one object pair, and the outliers are filtered out by eliminating the clusters with small sizes or authorities[3]. Cho et al.[1] and Zhang et al.[25] proposed two novel methods based on agglomerative clustering. Such methods are based on heuristic rules and therefore global optimum cannot be guaranteed. Other attempts perform clustering via mode-seeking in the graph domain. Liu et al.[17] introduced a graph shift algorithm to detect dense subgraphs with iterative shrinking and expansion. Jouili et al.[12] presented a median graph shift which is an extension of the medoid shift based on the concept of the median graph. Both methods perform mode-seeking by shifting from one subgraph to another subgraph, but not between nodes. As will be shown, such method largely depends on the initialization and is prone to local minima. Cho et al.[3] proposed a node-shifting scheme based on the high-order personalized PageRank (PPR) matrix. Its iterative PPR propagation scheme tends to accumulate errors on outliers, and PPR matrix is computationally expensive.

In this paper, we try to solve those challenges with a unified framework-Density Maximization. We first propose a density local estimator $(D L E)$ which is a reliable measure for the quality of matches. Our work is inspired by Lin et al.[16] who observed that the geometric transformations associated with neighbouring true matches are smoothly varying even for significant displacements. The basic idea of $D L E$ is to measure the quality of a match by using only the inliers from a local smooth neighbourhood, in order to avoid being cluttered by outliers and other object correspondences. Density Maximization is then modeled as maximization of the $D L E$ values both locally and globally. Our local maximization, named Density-Ascent Shift $(D A S)$, detects clusters of nodes as well as eliminates outliers. $D A S$ is a mode-seeking method similar to $[12,3,17]$, but is much more robust to clutter. Furthermore, $D A S$ is much faster than $[12,3,17]$ because it does not require iterations while [12, 3, 17] do. Our global maximization, called Density-Ascent Update $(D A U)$, refines the candidate matches by efficiently exploring a much larger matching space. $D A U$ is similar to the progression method of Cho et al.[5] which updates matches in a progressive way, but is more than one order of magnitude faster than [5] while introducing much less outliers.

Density Maximization performs $D A S$ and $D A U$ iteratively until convergence. At each iteration, the result of $D A S$ is the starting point of $D A U$. This simple scheme ensures that updating candidate matches is mainly based on the inliers, thus leading to a high precision. Similar to the progression method [5], Density Maximization is orthogonal to specific graph matching algorithms and can be used to improve any of them. Experimental evaluation on extensive natural images demonstrates that Density Maximization significantly boosts the true matches and enables graph matching to handle outliers and many-to-many object correspondences.

Compared to the state-of-the-art methods, Density Maximization has the following advantages:

(1) It addresses the three challenges of graph matching in a unified framework.

(2) It is much more robust to significant clutter.

(3) It is more than one order of magnitude faster.

(4) Its precision is much higher.

\section{Background}

\subsection{Graph matching formulation}

Let $G^{P}=\left(V^{P}, E^{P}, A^{P}\right)$ and $G^{Q}=\left(V^{Q}, E^{Q}, A^{Q}\right)$ be two attributed graphs, where $V$ denotes a set of nodes, $E$, edges, and $A$, attributes. The objective of graph matching is to find a mapping between $V^{P}$ and $V^{Q}$, represented by a binary assignment matrix $X \in\{0,1\}^{n^{P} \times n^{Q}}$ with $n^{P}$ and $n^{Q}$ denoting the numbers of nodes in $G^{P}$ and $G^{Q}$ respectively. $X_{i, a}=1$ implies that node $v_{i}^{P} \in V^{P}$ matches node $v_{a}^{Q} \in V^{Q}$. Let $x \in\{0,1\}^{n^{P} n^{Q}}$ denote the column-wise vectorized replica of $X$, the integer quadratic programming (IQP) formulates graph matching as

$$
x^{*}=\arg \max _{x} x^{T} W x,
$$

s.t. $\quad \forall i \quad \sum_{a=1}^{n^{Q}} x_{i a} \leq 1, \forall a \quad \sum_{i=1}^{n^{P}} x_{i a} \leq 1$ and
$x \in\{0,1\}^{n^{P} n^{Q}}$

The two-way constraints of (1) refer to the one-to-one matching from $G^{P}$ to $G^{Q}$. In sparse feature correspondence, the nodes represent features extracted from each image while the edges denote relationships between features. The symmetric affinity matrix $W$ encodes both the unary and pairwise similarities. A diagonal element $W_{i a ; i a}$ represents a unary similarity of a match $\left(v_{i}^{P}, v_{a}^{Q}\right)$, and a nondiagonal term $W_{i a ; j b}$ refers to a pairwise similarity of two matches $\left(v_{i}^{P}, v_{a}^{Q}\right)$ and $\left(v_{j}^{P}, v_{b}^{Q}\right)$. Every element of $W$ is non-negative. 


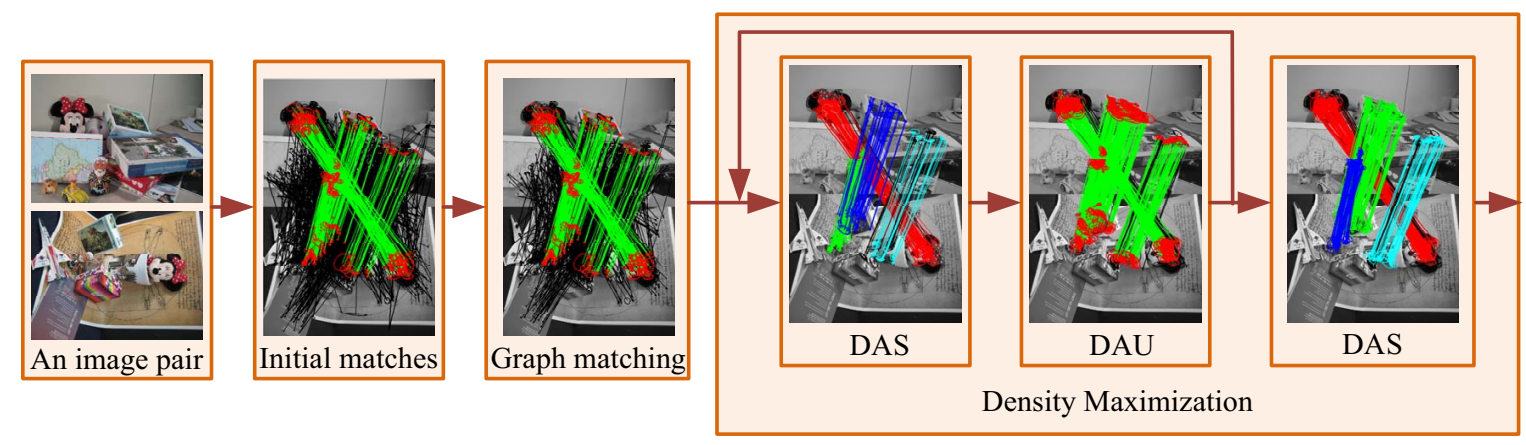

Figure 1. Overview of our Density Maximization framework. The Graph Matching result contains 283 true matches together with 315 outliers. Density Maximization improves Graph matching by iterating $D A S$ and $D A U$. DAS eliminates most outliers and detects four clusters of true matches. $D A U$ boosts the number of true matches to 416 and introduces only 63 outliers. The final step $D A S$ further removes 19 outliers. True matches are shown with color lines and false matches are shown with black lines.

\subsection{Analysis of IQP}

Aside from the NP-complete nature, IQP has several other limitations.

Firstly, the combinatorial nature makes the computation of $W$ intractable. A real-world image of common size contains more than $n=1000$ sparse features by using the popular affine or scale invariant detectors such as SIFT [18], MSER [20] and Harris Affine [21]. This results in a huge affinity matrix of dimension $(n \times n)^{2}=1000^{4}$. Most graph matching methods reduces the size of $W$ by using matches at a relatively high unary similarity. Such a simple scheme often leads to the loss of useful information hidden in the full matching space [5].

Secondly, IQP prefers more matches. Let $v_{i}^{P}$ and $v_{a}^{Q}$ denote two noisy features which have no true matching ones, setting $X_{i ; a}=1$ non-decreases the objective function $x^{T} W x$ because every element of $W$ is non-negative. This means that IQP prefers including the matches of all the features, even if they might be outliers. To alleviate this problem, some methods sparsify $x$ by increasing large values while smoothing out small values $[2,8,14,23,11]$. The results still contains many outliers.

Finally, IQP assumes that the feature set from one image belongs to a single cluster. This means that the quality of one match is measured based on all current matches. From (1) we can see that the contribution of each match $\left(v_{l}^{P}, v_{m}^{Q}\right)$ to the objective function is

$$
C(l, m)=x_{l m}\left(\sum_{i \neq l, a \neq m} W_{i a ; l m} x_{i a}+\sum_{j \neq l, b \neq m} W_{l m ; j b} x_{j b}\right)
$$

If $C(l, m)>C(l, s)$, IQP prefers $\left(v_{l}^{P}, v_{m}^{Q}\right)$ to $\left(v_{l}^{P}, v_{s}^{Q}\right)$, and vice versa. $C(l, m)$ contains the similarities between $\left(v_{l}^{P}, v_{m}^{Q}\right)$ and all the other matches. This measure is problematic for many-to-many object correspondences because the matches in one object correspondence might clutter those in others. To avoid this, each object correspondence should be considered independently.

\section{Density Maximization}

Similar to $[2,4,14,18]$, we construct an association graph $G^{a g}=\left(V^{a g}, E^{a g}, A^{a g}\right)$ based on the affinity matrix $W$. We take each candidate match $\left(v_{i}^{P}, v_{a}^{Q}\right)$ as a node $v_{i a} \in V^{a g}$, and its associated weight $W_{i a ; j b}$ as the attribute $a_{i a ; j b} \in A^{a g}$ of the edge $e_{i a ; j b} \in E^{a g}$. Then the original graph matching problem between $G^{P}$ and $G^{Q}$ becomes node selection problem in the graph $G^{a g}$. For brevity, we will use a single letter to index the node of $G^{a g}$ in the following sections, e.g., $v_{i}$ denotes the $i-t h$ node and $W_{i ; j}$ denotes the component of $W$ at the $i-t h$ row and the $j-t h$ column.

Figure 1 shows the framework of Density Maximization. Given an image pair, the salient features are firstly extracted from each image and then $N_{C}$ candidate matches are readily established using unary descriptors of the features at a relatively low cost. Those matches are taken as the nodes of an initial association graph. Graph matching selects best nodes from it in order to maximize the objective function in (1). Then a reduced set of nodes and their edges are selectively used to construct a new graph which is called valid graph $G^{V}$ in this paper. Density Maximization improves $G^{V}$ by iterating $D A S$ and $D A U$. Based on $G^{V}, D A S$ finds the clusters of nodes as well as removes the outliers by local maximization of the $D L E$ values. $D A U$ produces a updated graph $G^{U}$ with $N_{C}$ nodes by global maximization of the $D L E$ values via exploring a much larger graph called potential graph $G^{T}$. At each iteration, the result of $D A S$ is the starting point of $D A U$. This ensures that the updates of matches are mainly based on inliers. The iterations continue until the total $D L E$ values no longer increase. The final step $D A S$ further removes the outliers introduced by $D A U$. As can be seen from Fig.1, Density Maximization is orthogonal to specific graph matching algorithms, and any 


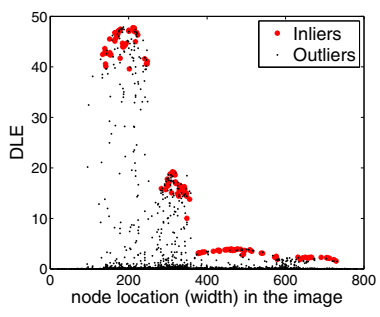

(a)

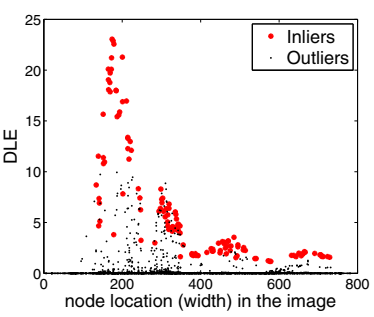

(b)
Figure 2. Kernel density estimation for inliers (denoted by red star) and outliers (denoted by black dot). (a) No $\Omega$ constraint. (b) With $\Omega$ constraint.

of them can be adopted as the graph matching module in the framework.

\subsection{Density local estimator}

Recently, graph density has shown its potentials to identify inliers and detect strongly connected node clusters in an association graph. A few attempts to define the graph density include the average kernel density of Liu et al.[17], the random walk density of Cho et al.[4], and the personalized PageRank density of Cho et al.[3]. Now we define our density local estimator $(D L E)$. The main difference between $D L E$ and other methods lies in its well-defined local smooth domain.

The intuitive of $D L E$ is to estimate graph density at one node by using only the nodes within a same object, so that it can avoid the clutter problem introduced by outliers and the nodes in other objects. However, it is difficult to determine whether two nodes belong to a same object. Fortunately, it has been observed that the geometric transformations associated with neighbouring matches in a same object are smoothly varying even for significant displacements [16]. This reveals a simple method to approximately identify the nodes within a same object by using a local smooth neighbourhood $\Omega . \Omega(i)$ should satisfy two criteria: (1) Locality: the neighbours are within a close proximity to node $v_{i}$. (2) Smoothness: the neighbours should have similar values to $v_{i}$ for a same measure. These criteria prevent the scope of neighbours from extending into outliers and the nodes in other objects.

We adopt the popular kernel density estimation method to compute the graph density locally. We consider node selection as a distribution and use $x_{i}$ to denote the probability of selecting node $v_{i}$. Suppose we sample the distribution $N(N \rightarrow \infty)$ times, then the number of selecting $v_{i}$ is $N x_{i}$. The density at $v_{i}$ is

$$
D L E(i)=\frac{\sum_{j \in \Omega(i)} N x_{j} K(i, j)}{N}=\sum_{j \in \Omega(i)} x_{j} K(i, j)
$$

This is called $D L E$ in this paper. $K(i, j)=W_{i ; j}$ implies

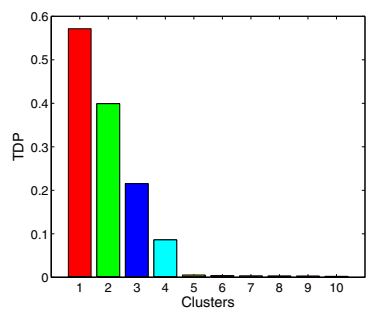

(a)

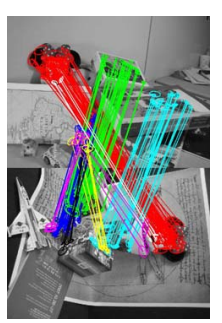

(b)
Figure 3. (a)Top 10 max TDP values. (b)The clusters for top 10 max TDP values. The match clusters of the four object pairs (denoted with red, green, blue and cyan lines) have TDP values significantly larger than those of the false match clusters.

the similarity between $v_{i}$ and $v_{j}$. The only difference between $D L E$ and the classical kernel density estimation lies in $\Omega$.

The Locality of $v_{j}$ with respect to $v_{i}$ is defined by using the k-nearest neighbour function $k N N(\cdot, k)$ :

$$
L\left(v_{i}, v_{j}\right)=1 \quad \text { if } \quad v_{j} \in k N N\left(v_{i}, k\right), \quad 0 \quad \text { otherwise. }
$$

The Smoothness of $\Omega(i)$ is defined on two measures: the geometric transformation and the probability of node selection. The Smoothness of geometric transformation between node $v_{i}$ and $v_{j}$ amounts to $W_{i ; j}$. The Smoothness of probability is measured by $\exp \left(-\left(x_{i}-x_{j}\right)^{2} / \sigma^{2}\right)$ with a parameter $\sigma$. Then $\Omega(i)$ is defined as a $\varepsilon-$ neighbourhood

$$
\Omega(i)=\left\{v_{j} \in V^{V} \mid \Phi(i, j)>\varepsilon\right\} \bigcup\left\{v_{i}\right\}
$$

where $\Phi(i, j)=L\left(v_{i}, v_{j}\right) W_{i, j} \exp \left(-\left(x_{i}-x_{j}\right)^{2} / \sigma^{2}\right)$ and the parameter $\varepsilon$ controls the size of $\Omega(i)$. Figure 2 demonstrates the impact of $\Omega$. With the constraint of $\Omega$, the inliers' $D L E$ values almost consistently larger than those of outliers at nearby locations.

The node selection probability $x$ naturally corresponds to the solutions to (1) since many graph matching methods solve (1) by relaxing the constraints on $x$ such that its elements can take real values in $[0,1]$. In those methods, $x$ can be viewed as the confidence that the matches are true [14] or as the probability of visits by random walks $[2,13,4]$. In this paper we consider $x$ as the node selection probability. For other graph matching methods in which $x$ are integer, we normalize $x$ to obtain a uniform distribution. Therefore $D L E$ is orthogonal to specific graph matching algorithms whether $x$ are continuous or not, unlike other methods.

\subsection{Density Ascent Shift}

As shown in Fig.1, the aim of $D A S$ is to produce node clusters and eliminate outliers from valid graph $G^{V}$. DAS is a mode-seeking method and the density modes on a graph are defined as follows. 


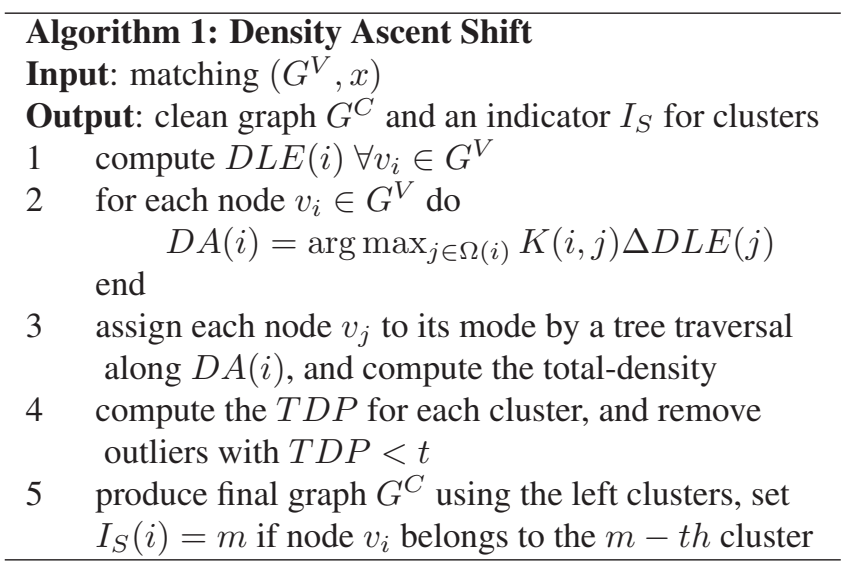

Definition 1 Density modes on a graph are local maximizers of the $D L E$ values.

$D A S$ performs mode-seeking along the density-ascent direction. The density-ascent $D A(i)$ of node $v_{i}$ is formulated as

$$
D A(i)=\arg \max _{j \in \Omega(i)} K(i, j) \Delta D L E(j)
$$

which means the neighbouring node of $v_{i}$ with the highest expectation of $D L E$ increment. This density-ascent is the steepest ascent over the $D L E$ values within $\Omega(i) . \Omega(i)$ prevents shifting into irrelevant clusters. Similar to other mode-seeking methods[12, 4, 3], DAS is guaranteed to converge.

Theorem 1 A finite sequence of density-ascent shifts from any node converges to a density mode.

Proof Since $\Omega(i)$ of any node $v_{i}$ includes itself, the $D L E$ values of a sequence of shifts keep strictly increasing until the shifts reach a node whose density-ascent is itself. The final node, therefore, is the density mode, and the length of the sequence is $\left|V^{V}\right|$ at most, with $\left|V^{V}\right|$ denoting the number of nodes in $G^{V}$.

For each node, we compute its density-ascent just once. Then the successive density-ascent for any node already exists. The trajectory of nodes sharing a common density mode builds a tree, and leads to a natural cluster. Then the cluster label of all nodes associated with each disjoint tree can be assigned in a single tree traversal, similar to the medoid shift[22].

We define the total-density of each cluster as the sum of the $D L E$ values of its members, and the total-densitypercentage $(T D P)$ of each cluster as the ratio between its total-density and the sum of the total-densities of all the clusters. The clusters of outliers usually have very small total-density, so that $T D P$ provides a reliable measure for detection and elimination of them, as shown in Fig.3. DAS is depicted in Algorithm 1.

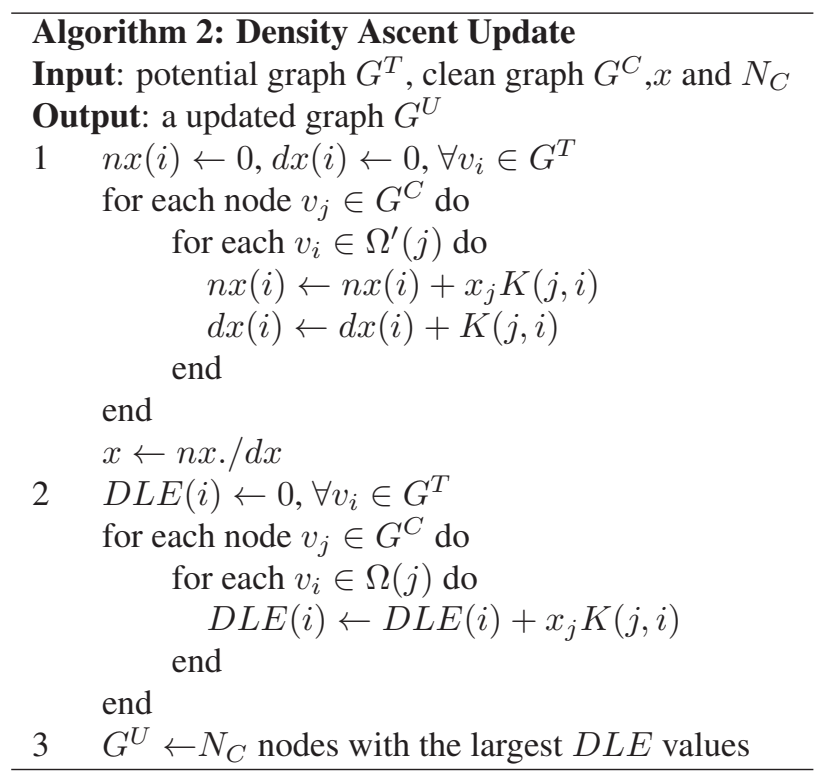

\subsection{Density Ascent Update}

Given a clean graph $G^{C}$, the aim of $D A U$ is to produce a updated graph $G^{U}$ with $N_{C}$ nodes by maximizing the total $D L E$ values. To achieve this, $D A U$ explores the potential graph $G^{T}$ which contains $G^{C}$ but is much larger. $G^{T}$ covers most true matches and will be detailed later. $D A U$ firstly estimates the $D L E$ values of the nodes in $G^{T}$, and then select $N_{C}$ best nodes with largest values to construct $G^{U}$. since $G^{C} \subset G^{T}$, this global maximization scheme ensures that $D A U$ non-decreases the total $D L E$ values.

To compute the $D L E$ value for each node $v_{i}$ in $G^{T}$, we need to identify $\Omega(i)$ at first. But the node selection probability $x_{i}$ might be unavailable if $v_{i}$ does not belong to $G^{C}$. Here we approximate $x_{i}$ by

$$
x_{i}=\frac{\sum_{j \in \Omega^{\prime}(i)} x_{j} K(i, j)}{\sum_{j \in \Omega^{\prime}(i)} K(i, j)}
$$

which is a weighted average of the selection probabilities over a local smooth neighbourhood $\Omega^{\prime}(i) . \quad \Omega^{\prime}(i)$ is similar to $\Omega(i)$ but does not consider the probability Smoothness since $x_{i}$ is unknown. However, $x_{j}$ for $j \in \Omega^{\prime}(i)$ might be unavailable. We observe that $\Omega^{\prime}(i)$ is nearly symmetric for true matches. By investigating the nodes for true matches in Fig.1 we find that if $j \in \Omega^{\prime}(i)$ the probability for $i \in \Omega^{\prime}(j)$ is above $90 \%$. Therefore (7) can be approximately rewritten as $x_{i}=\sum_{i \in \Omega^{\prime}(j)} x_{j} K(j, i) / \sum_{i \in \Omega^{\prime}(j)} K(j, i)$. Let $n x(i)=$ $\sum_{i \in \Omega^{\prime}(j)} x_{j} K(j, i)$ and $d x(i)=\sum_{i \in \Omega^{\prime}(j)} K(j, i)$, the contribution of each node $v_{j}$ in $G^{C}$ to $n x(i)$ is $x_{j} K(j, i)$, and that to $d x(i)$ is $K(j, i)$ if $i \in \Omega^{\prime}(j)$. Therefore all $x_{i}$ can be estimated very efficiently by traversing the nodes of $G^{C}$.

Since $\Omega^{\prime}(i)$ is nearly symmetric, $\Omega(i)$ is also nearly symmetric because $\exp \left(-\left(x_{i}-x_{j}\right)^{2} / \sigma^{2}\right)$ is symmetric. Then 
(3) can be rewritten as $D L E(i)=\sum_{i \in \Omega(j)} x_{j} K(j, i)$ which means that the contribution of each node $v_{j}$ in $G^{C}$ to $D L E(i)$ is $x_{j} K(j, i)$. Therefore all $D L E(i)$ can be efficiently calculated by traversing the nodes of $G^{C}$. DAU is summarized in Algorithm 2.

The potential graph $G^{T}$ is constructed using $Z$ matches for each feature based on the unary similarity. We test on the image pairs of the intra-class dataset[1] which own large intra-category variations, and find that $G^{T}$ covers more than $95 \%$ true matches when $Z=40$. This reveals that exploring the whole matching space like Cho et al.[5] is unnecessary.

\subsection{Analysis}

Using the approximate nearest neighbour (ANN) search, the computational complexity of $D A U$ is $O\left(k\left|V^{V}\right| \log \left(Z n^{P}\right)\right)$ with $\left|V^{V}\right|$ denoting the node number of $G^{V}, n^{P}$ denoting the node number of graph $G^{P}$ (i.e., the feature number of one image) and $k=50$. As far as we know, the only work similar to $D A U$ is the progression method[5] whose computational complexity is $O\left(k_{1} k_{2}\left|V^{V}\right| \log \left(n^{P}\right) \log \left(n^{Q}\right)\right)$ with $k_{1}=25$ and $k_{2}=5$. $D A U$ is more than one order of magnitude faster because $k_{1} k_{2} \log \left(n^{P}\right) \log \left(n^{Q}\right) / k \log \left(Z n^{P}\right)>10$ for general cases with $n^{P}>1000$ and $n^{Q}>1000$. The main difference is that $D A U$ explores the potential graph $G^{T}$ while the progression method searches the whole matching space based on $G^{V}$. Since $G^{T}$ covers most true matches, exploring $G^{T}$ does not degrade the performance. On the other hand, this scheme successfully avoids many outliers in the whole matching space, as will be shown in the experiments.

The computational complexity of DAS is $O\left(k\left|V^{V}\right| \log \left|V^{V}\right|\right)$, more than one order of magnitude faster than most mode-seeking methods. The high efficiency benefits from its non-iteration scheme. More importantly, either $D A U$ or $D A S$ is much faster than most graph matching methods $[2,8,14,15,23,19,11]$, indicating that we can improve graph matching without introducing too much computational cost.

An important trick of our Density Maximization framework is that $D A U$ takes the result of $D A S$ as its starting point at each iteration. This ensures that the updating matches is mainly based on inliers. In this way, Density Maximization significantly increases the precision in sharp contrast with the progression method, as shown in Fig.4.

\section{Experiments}

In our experiments, the candidate matches are generated using the SIFT descriptor. To measure the similarity between two matches $\left(v_{i}^{P}, v_{a}^{Q}\right)$ and $\left(v_{j}^{P}, v_{b}^{Q}\right)$, we adopted the symmetric transfer error $d(i a ; j b)$ used in $[5,13,1,4]$. The affinity matrix $W$ is calculated by $W_{i a ; i b}=\max (50-$ $d(i a ; j b), 0)$. In Density Maximization, we set $\sigma=0.2$, $k=50, \varepsilon=10$ and $t=0.03$.

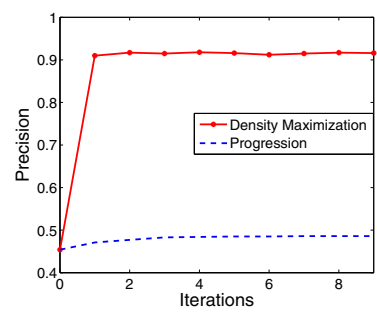

Figure 4. Performance growth on the image pair in Fig.1 by our Density Maximization and the progression method[5]. The plot shows the precision w.r.t the iteration steps. Note that the step 0 denotes the result by graph matching.

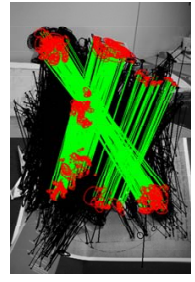

(a)

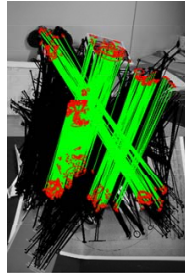

(b)

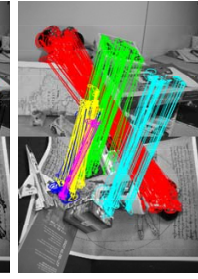

(c)

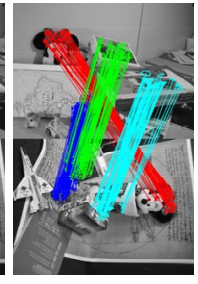

(d)
Figure 5. (a)The result by GP[5] based on the graph matching result in Fig.1. (b)The result by our $D A U$. (c)The result by GP together with our $D A S$. The false match clusters are denoted by yellow and magenta lines. (d)The result by our $D A U$ together with our $D A S$. There is no false match clusters.

We test Density Maximization on three challenging benchmark datasets: Intra-class dataset[1], ETHZ toys dataset[10], Co-recognition dataset[6]. Intra-class dataset consists of 30 image pairs of large transformations and intra-class variation. It provides detected MSER features and initial matches. In this dataset, most images have a small number of features and only several hundreds of initial matches. For fair comparison, we adopt those features and always fix the number of candidate matches $N_{C}$ to the same as the number of the given initial matches. ETHZ toys dataset includes 9 different rigid/non-rigid objects together with the test images of significant clutter. Co-recognition dataset contains 6 image pairs with complex many-to-many object correspondences. The ground truth feature correpondences are manually constructed for each image pairs to enable quantitatively evaluation. For these two dataset, we use the MSER and the Harris affine detectors with the SIFT descriptor, and set $N_{C}=3000$. Our testing environment is MS Windows 7 Professional with Intel Core i5-3550 CPU 3.3GHz, 16GB RAM.

\subsection{Density Maximization vs related work}

Density Maximization contains novel approaches to both updating matches (i.e., $D A U$ ) and clustering matches (i.e., $D A S)$. We will show the effectiveness of it in both of these 


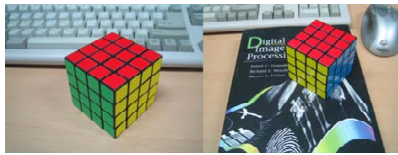

(a)

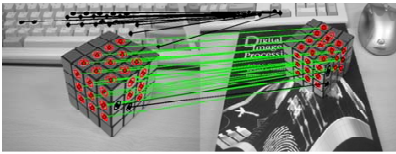

(c)

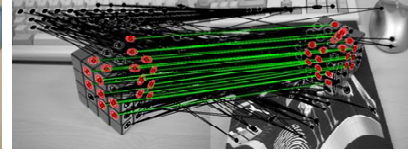

(b)

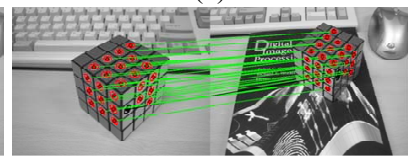

(d)
Figure 6. (a)A image pair. (b)The result by SAE[17]. (c)The result by (ACC)[1]. (d)The result by our $D A S$. True matches are shown with green lines and false matches are shown with black lines.

\begin{tabular}{c|cc|ccc|cc}
\hline D & PG & $D A U$ & ACC & SAE & $D A S$ & PG+ & DM \\
& & & & & & ACC & \\
\hline \hline 1 & 81 & 83 & 71 & 43 & 83 & $71 / 70$ & $73 / 81$ \\
\hline 2 & 69 & 77 & 63 & No & 85 & $62 / 69$ & $72 / 88$ \\
\hline 3 & 66 & 81 & 67 & No & 91 & $61 / 74$ & $75 / 92$ \\
\hline
\end{tabular}

Table 1. Recall (\%) of PG and our $D A U$, Precision (\%) of ACC, SAE and our DAS, Recall/Precision (\%) of PG+ACC and our Density Maximization (DM). D 1,2 and 3 denote Intra-class dataset, ETHZ toys dataset and Co-recognition dataset respectively. 'No' denotes the failure of SAE.

steps as well as a whole.

Firstly we compare our $D A U$ with the graph progression (GP)[5] since it is the only similar work to $D A U$ as far as we know. For fair comparison, we adopt the same progressive framework as GP, which performs graph matching and updating matches iteratively. Since the aims of both $D A U$ and GP are to boost the true matches, we access Recall on the three datasets. The results of GP contain lots of overlapping matches. To compute Recall more accurately, we count the overlapping matches only once. The overall results are given in Table 1. Compared to our $D A U, \mathrm{GP}$ tends to introduce more outliers which are very difficult to remove. Figure 5 shows the outliers of an example. The average times for each iteration of GP to process each image pair in the three data-sets are $0.45,5.32$ and 31.53 seconds respectively. The corresponding times for $D A U$ are 0.27 , 1.64 and 3.73 seconds respectively.

Secondly, we compare our $D A S$ with two state-of-thearts methods: the agglomerative correspondence clustering (ACC)[1] and the Shrink-and-Expansion (SAE)[17]. Since SAE cannot handle both ETHZ toys and Co-recognition datasets (the source code provided by the authors on the internet has 'out of memory' problem when handling thousands of matches), we only report its result for Intra-class dataset. Since the aim of $D A S$ is to improve Precision, we access Precision on the three datasets. The overall results are given in Table 1 and an example is shown in Fig.6. SAE

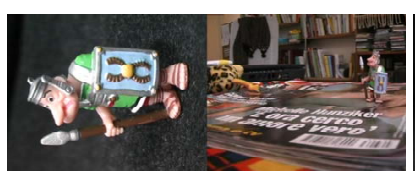

(a)

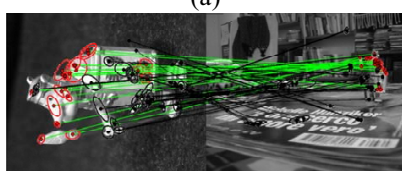

(c)

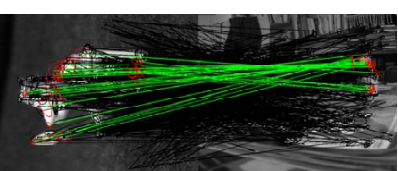

(b)

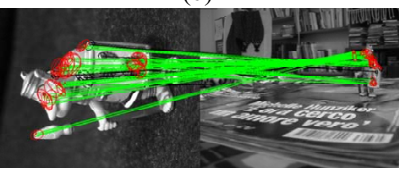

(d)
Figure 7. (a)A image pair. (b)Graph matching result. (c)The result by GP+ACC. (d)The result by our Density Maximization. True matches are shown with green lines and false matches are shown with black lines.

\begin{tabular}{l|lllll}
\hline D & SM & PM & BGM & IPFP & RRWM \\
\hline \hline 1 & $43 / 47$ & $39 / 44$ & $32 / 36$ & $47 / 39$ & $45 / 41$ \\
\hline 2 & $23 / 64$ & $27 / 52$ & $18 / 45$ & $33 / 67$ & $29 / 53$ \\
\hline 3 & $27 / 51$ & $25 / 49$ & $21 / 42$ & $33 / 41$ & $31 / 44$ \\
\hline
\end{tabular}

Table 2. Performance improvement (\%) over Recall/Precision by Density Maximization.

tends to include lots of outliers. The results of ACC are much better, but are still noisy. In contrast, our $D A S$ successfully detects true matches and distinguishes them from outliers. To process each image pair in the three datasets $D A S$ takes only $0.69,1.72$ and 1.93 seconds on average, while ACC takes 8.74, 78.52 and 95.73 seconds. SAE is much slower and takes more than one minute on average to process each image pair of Intra-class dataset.

Finally, we compare our Density Maximization with a combined method-GP+ACC. GP+ACC is performed in a similar way of Density Maximization: GP and ACC are performed iteratively till convergence. We measure both Recall and Precision on the three datasets. As shown in Fig.7, the outliers introduced by GP cannot be eliminated by ACC, and result in noisy clusters. So GP+ACC increases Recall at the expense of Precision. Our Density Maximization solves this problem effectively by avoiding outliers from source. It largely outperforms $\mathrm{GP}+\mathrm{ACC}$ in both precision and recall as shown by Table 1 .

\subsection{Density Maximization vs Graph Matching}

In this experiment, we show the improvement of Density Maximization on several state-of-the arts graph matching methods: SM[14], PM[24], BGM[8], IPFP[15] and RRWM[2]. The quantitative results are summarized in Table 2, and some examples are shown in Fig.8. The graph matching methods cannot distinguish inliers from outliers, and fail to separate matches of one object from those of others. Density Maximization solves these problems effectively by detecting clusters of true matches. The precision 


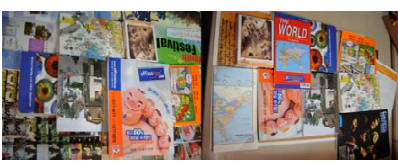

(a)

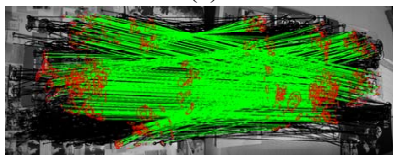

(c)

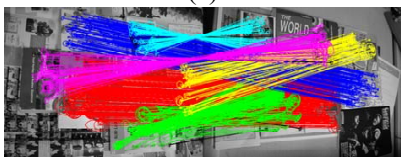

(e)

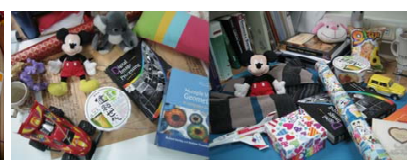

(b)

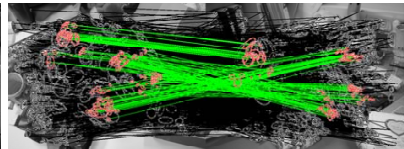

(d)

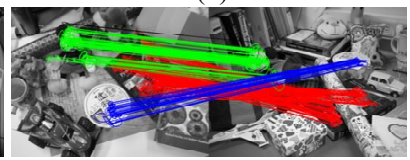

(f)
Figure 8. (a) and (b) are two input image pairs. (c)Result by RRWM[2] for (a). (d)Result by SM[2] for (b). (e)The result by our Density Maximization with RRWM as the graph matching module. (f)The result by our Density Maximization with SM as the graph matching module. True matches are shown with color lines and false matches are shown with black lines.

is boosted by $36 \% \sim 67 \%$, and the recall by $18 \% \sim 47 \%$.

\section{Conclusion}

We introduced a unified framework, called Density Maximization, which effectively resolves the three limitations of conventional graph matching and achieves impressive performance improvement. By globally and locally maximizing a novel proposed density estimator, i.e., density local estimator, Density Maximization leads to the integration of updating matches, eliminating outliers and cluster detection. We point out that the key to the high performance is twofold: a well-defined local smooth neighbourhood to avoid clutter and an iteration scheme to ensure that updating matches is mainly based on inliers. Experiments demonstrate that Density Maximization is adequate for very challenging real-world images which contain many-to-many object correspondences and significant outliers.

\section{Acknowledgement}

This work is supported by Australian Research Council (ARC) Linkage Grant LP0991757.

\section{References}

[1] M. Cho, J. Lee, and K. M. Lee. Feature correspondence and deformable object matching via agglomerative correspondence clustering. ICCV, 2009.

[2] M. Cho, J. Lee, and K. M. Lee. Reweighted random walks for graph matching. ECCV, 2010.

[3] M. Cho and K. M. Lee. Authority-shift clustering: Hierarchical clustering by authority seeking on graphs. CVPR, 2010.
[4] M. Cho and K. M. Lee. Mode-seeking on graphs via randomwalks. CVPR, 2012.

[5] M. Cho and K. M. Lee. Progressive graph matching: Making a move of graphs via probabilistic voting. CVPR, 2012.

[6] M. Cho, Y. M. Shin, and K. M. Lee. Co-recognition of image pairs by data-driven monte carlo image exploration. ECCV, 2008.

[7] D. Conte, P. Foggia, C. Sansone, and M. Vento. Thirty years of graph matching in pattern recognition. IJPRAI, pages 265-298, 2004.

[8] T. Cour, P. Srinivasan, and J. Shi. Balanced graph matching. NIPS, 2007.

[9] O. Duchenne, F. Bach, I. Kweon, and J. Ponce. A tensorbased algorithm for high-order graph matching. CVPR, 2009.

[10] V. Ferrari, T. Tuytelaars, and L. V. Gool. Simultaneous object recognition and segmentation from single or multiple model views. IJCV, 67(2):159-188, 2006.

[11] M. Gori, M. Maggini, and L. Sarti. Exact and approximate graph matching using random walks. TPAMI, 27(7):11001111, 2005.

[12] S. Jouili, S. Tabbone, and V. Lacroix. Median graph shift: A new clustering algorithm for graph domain. ICPR, 2010.

[13] J. Lee, M. Cho, and K. M. Lee. Hyper-graph matching via reweighted random walks. CVPR, 2011.

[14] M. Leordeanu and M. Hebert. A spectral technique for correspondence problems using pairwise constraints. ICCV, 2005.

[15] M. Leordeanu and M. Herbert. An integer projected fixed point method for graph matching and map inference. NIPS, 2009.

[16] W. Lin, S. Liu, Y. Matsushita, and T. Ng. Smoothly varying affine stitching. CVPR, 2011.

[17] H. Liu, L. J. Latecki, and S. Yan. Fast detection of dense subgraph with iterative shrinking and expansion. TPAMI, 2013.

[18] D. G. Lowe. Object recognition from local scale-invariant features. ICCV, 1999.

[19] J. Maciel and J. Costeira. A global solution to sparse correspondence problems. TPAMI, 25(2):187-199, 2003.

[20] J. Matas, O. Chum, M. Urban, and T. Pajdla. Robust wide baseline stereo from maximally stable extremal regions. BMVC, 2002.

[21] K. Mikolajczyk and C. Schmid. Scale and affine invariant interest point detectors. IJCV, 2004.

[22] Y. A. Sheikh, E. A. Khan, and T. Kanade. Mode-seeking by medoid shifts. ICCV, 2007.

[23] L. Torresani, V. Kolmogorov, and C. Rother. Feature correspondence via graph matching: Models and global optimization. ECCV, 2008

[24] R. Zass and A. Shashua. Probabilistic graph and hypergraph matching. CVPR, 2008.

[25] W. Zhang, X. Wang, D. Zhao, and X. Tang. Graph degree linkage: agglomerative clustering on a directed graph. ECCV, 2012. 